\title{
Health Care: Diseases diagnosis Improvement using Optimization Techniques
}

\author{
Harman Ali \\ M. Tech. Scholar \\ Department of CSE, BIRT, Bhopal (M.P) India
}

\author{
Imran Ali Khan \\ Assistant Professor \\ Department of CSE, BIRT, Bhopal (M.P) India
}

\begin{abstract}
Health is a potential state in which someone performs well, both mentally and physically living within one inhabits environment. In this paper we proposed a new model for the improving the health care and diagnosis system using machine learning techniques, classification results of data which is available in the form of big data, here we measured the classification rate of various patients and related diseases diagnosis system for the improving the rate of early detection of any diseases at early stages.
\end{abstract}

\section{Keywords}

Health Care, Data Mining, Machine learning, CDSS

\section{INTRODUCTION}

Modern medicine is a unique synthesis of a patient, healthcare professionals, and technology. For the past 10-15 years there has been a slow but steady increase in the use and storage of electronic machine readable formats known as electronic health records (EHRs). It is unlikely that there will be major improvements in the quality and cost of care, solely from the use of EHRs without the proper implementation and use of clinical decision support [8].

A clinical decision support system (CDSS) can be defined as "software that is designed to be of direct aid to clinical decision making in which the characteristics of an individual patient are matched to a computerized clinical knowledge base, and patient-specific assessments or recommendations are then presented to the clinician and/or the patient for a decision [13].

Another way to detach clinical knowledge from the internal programming language control code of the CDSS is through rule-based reasoning systems (also referred to as an expert system). Rules represent and manipulate knowledge in a declarative manner [13].

Over the last several decades numerous representations and systems have spawned but essentially all of them are expressed as IF THEN statements containing two parts: the conditions and the actions.

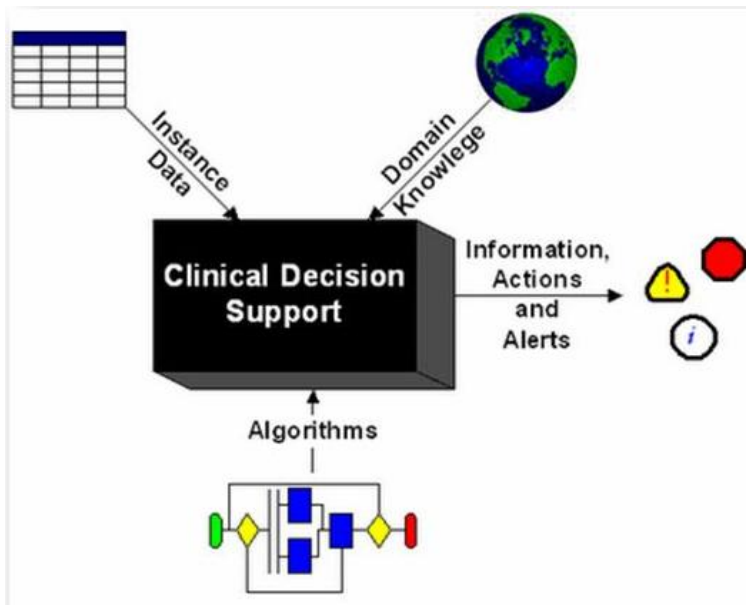

Figure-1: Principle Elements of a Clinical Decision Support System

There are various tools and technology for the prediction and classification re available machine learning is most popular tools among them. Machine learning is popular programming tools for the prediction and classification of large or huge amount of data, the classification is also a methods of data mining which primary object is to grouping of the same data or the same pattern of the data in a dingle group. Machine learning is basically differ from the traditional programming language.

\section{Traditional Programming}

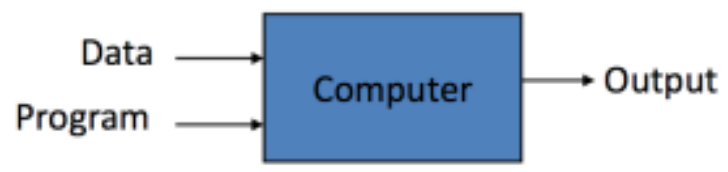

\section{Machine Learning}

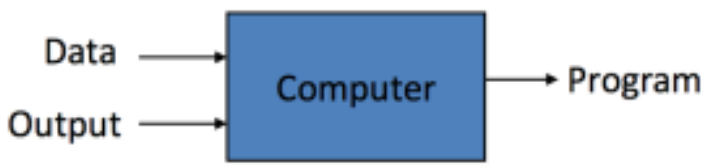

Figure 2: Machine learning Categories.

\section{RULE-BASED REASONING}

Another way to detach clinical knowledge from the internal programming language control code of the CDSS is through rule-based reasoning systems (also referred to as an expert system). Rules represent and manipulate knowledge in a declarative manner [13]. Over the last several decades numerous representations and systems have spawned but 
essentially all of them are expressed as IF THEN statements containing two parts: the conditions and the actions. In the mathematical sense a rule is in the form $\mathrm{A}=\mathrm{C}$, where $\mathrm{A}$ is the set of conditions or the antecedent, and $\mathrm{C}$ is the set of actions or the consequent. Rules allow the declarative expression of firstorder logic in an unambiguous, human readable form, at the same time retaining machine interpretability. Unlike the actions in workflow-driven CDSS, rules-based systems execute actions only in response to changes in the facts available to the rulesengine. There are two methods of processing rules for a rulebased system: Forward Chaining and Backward Chaining.

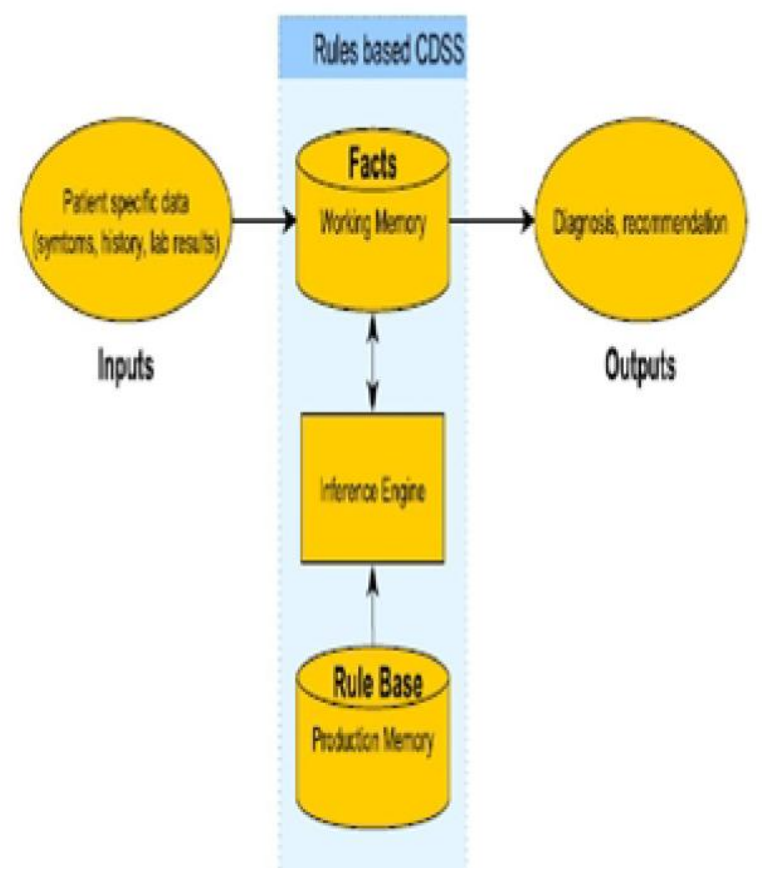

Figure 3: Generic model of a rule-based reasoning CDSS.

The rest of this paper is organized as follows In section II we discuss about the proposed methods and architecture. In section III we discuss about the empirical results study and their analysis. And finally conclude the paper.

\section{RELATED WORK}

[1] In this paper, author described the health care for the various diseases using machine learning algorithm over big data analytics. They proposed model for the various diseases diagnosis over the big data, big data which is a combination of variety, volume, velocity etc. here the big data analytics provide huge data for the medical science. They provide the model and do their experimental work on the big data of health care sector using machine learning techniques.

[2] In this article it is explained that asthma is one of the most prevalent and costly chronic conditions in the United States, which cannot be cured. However, accurate and timely surveillance data could allow for timely and targeted interventions at the community or individual level. Current national asthma disease surveillance systems can have data availability lags of up to two weeks. Rapid progress has been made in gathering non-traditional, digital information to perform disease surveillance. We introduce a novel method of using multiple data sources for predicting the number of asthma-related emergency department (ED) visits in a specific area. Our preliminary findings show that our model can predict the number of asthma $\mathrm{ED}$ visits based on near-real-time environmental and social media data with approximately $70 \%$ precision. The results can be helpful for public health surveillance, ED preparedness, and targeted patient interventions.

[3] In this paper, author illustrated the idea that the purely phenomenological knowledge that we can extract by analyzing large amounts of data can be useful in healthcare seems to contradict the desire of VPH researchers to build detailed mechanistic models for individual patients. But in practice no model is ever entirely phenomenological or entirely mechanistic. We propose in this position paper that big data analytics can be successfully combined with VPH technologies to produce robust and effective in silico medicine solutions. These domain-specific requirements suggest a need for targeted funding, in which big data technologies for in-silico medicine becomes the research priority.

[5] This paper represents the advances in information technology have witnessed great progress on healthcare technologies in various domains nowadays. However, these new technologies have also made healthcare data not only much bigger but also much more difficult to handle and process. To provide a more convenient service and environment of healthcare, this paper proposes a cyber-physical system for patient-centric healthcare applications and services, called Health-CPS, built on cloud and big data analytics technologies. The results of this study show that the technologies of cloud and big data can be used to enhance the performance of the healthcare system so that humans can then enjoy various smart healthcare applications and services.

[8] In this paper, they provide an introduction to machine learning tasks that address important problems in genomic medicine. Here, we describe how machine learning can be used to solve key problems in genomic medicine. Genomics is the study of the function and information structure encoded in the DNA sequences of living cells, whereas precision medicine is the practice of tailoring treatment based on all relevant information about the patient, including the patient's genome.

[9] This article describes that with a massive influx of multimodality data, the role of data analytics in health informatics has grown rapidly in the last decade. Deep learning, a technique with its foundation in artificial neural networks, is emerging in recent years as a powerful tool for machine learning, promising to reshape the future of artificial intelligence. Rapid improvements in computational power, fast data storage, and parallelization have also contributed to the rapid uptake of the technology The paper mainly focuses on key applications of deep learning in the fields of translational bioinformatics, medical imaging, pervasive sensing, medical informatics, and public health.

[12] This article presents a comprehensive overview of the challenges, pipeline, techniques, and future directions for computational health, the rapid growth of novel technologies has led to a significant increase of digital health data in recent years. More medical discoveries and new technologies such as mobile apps, capturing devices, novel sensors, and wearable technology have contributed to additional data sources. Most popular surveys of big data in health informatics have concentrated on biomedical aspects of big data, while a smaller percentage of papers focus on the computational perspective.

[17] The author justifies that the considerable growing of cardiovascular disease and its effects and complications as well as the high costs on society makes medical community seek for solutions to prevention, early identification and effective 
treatment with lower costs. Thus, valuable knowledge can be established by using artificial intelligence and data mining; the discovered knowledge makes improve the quality of service. Until now, different researches have been carried out in order to predict heart disease based on data mining methods such as classification and clustering methods; however, what has been less noticed is the exact diagnosis of disease with the lowest cost and time.

\section{PROPOSED METHOD}

In this section we discussed about the proposed new model for the health care system to compute some performance parameters such as accuracy using classification and optimization techniques. Here we improve the efficiency rate in the terms of accuracy for the proposed system compare than existing system which is provide better results in the medical science domain.

There are various classifier are available for the purpose of classify the data, support vector machine is one of among them, it is a set of classifier which classify the data on the given input data which is generated from the same pattern and also known as vector data, and then directly supported for the machine. Support vector machine is basically reduced the gap or margin between the class of data and hyper-plane of the data. They create the one or more class categories.

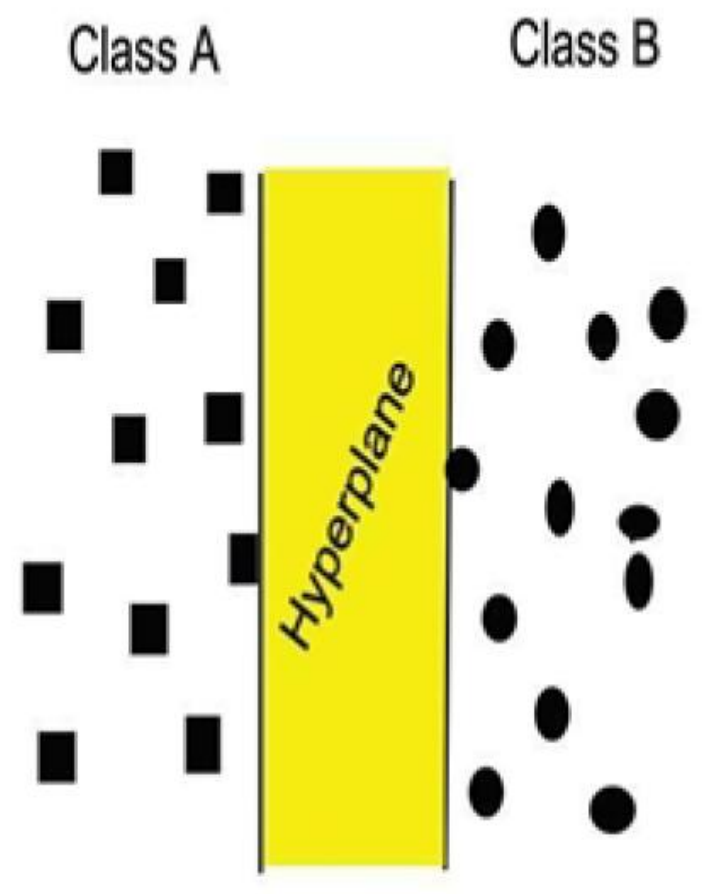

Figure 4: The SVM Class Data

begin

$\mathrm{t}=0$;

initialize particles $\mathrm{p}(\mathrm{t})$;

evaluate particles $\mathrm{p}(\mathrm{t})$;

while (termination conditions are unsatisfied)

begin

$\mathrm{t}=\mathrm{t}+1$;

update weights

select pbest for each particle

\author{
select gbest from $\mathrm{p}(\mathrm{t}-1)$; \\ calculate particle velocity $\mathrm{p}(\mathrm{t})$ \\ calculate particle position $\mathrm{p}(\mathrm{t})$ \\ evaluate particles $\mathrm{p}(\mathrm{t})$ \\ end \\ end
}

\section{EXPERIMENTAL RESULT ANALYSIS}

In this section, we show experimental process of that the comparative result analysis study for the Health care sector with disease diagnosis of various dataset such as Heart, Liver, Cancer etc. are performed. This process of disease diagnosis of various dataset is done by using Three methods that are Decision tree, SVM and Proposed method.

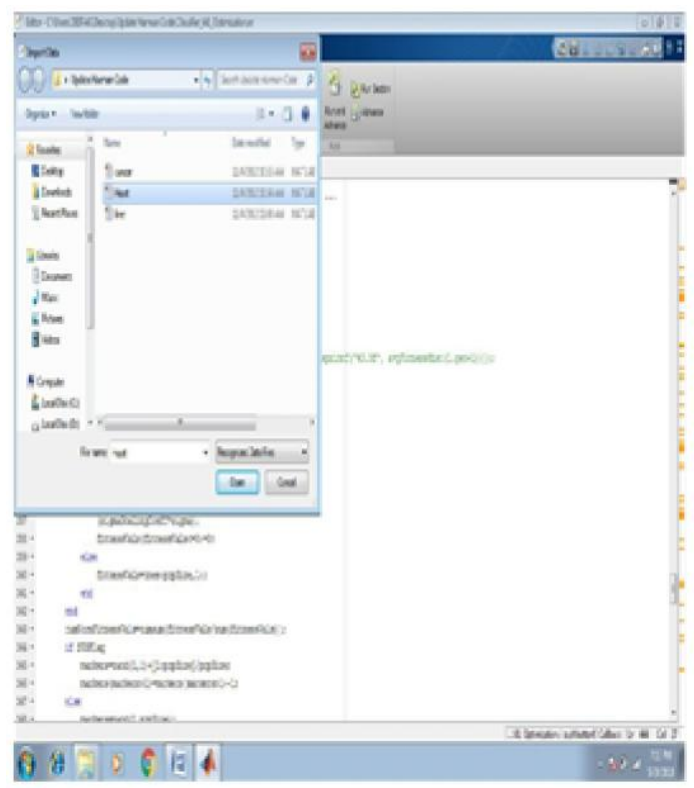

Figure-5: This windows show that the selection the Heart dataset for the selection of Decision Tree methods in the experimental process

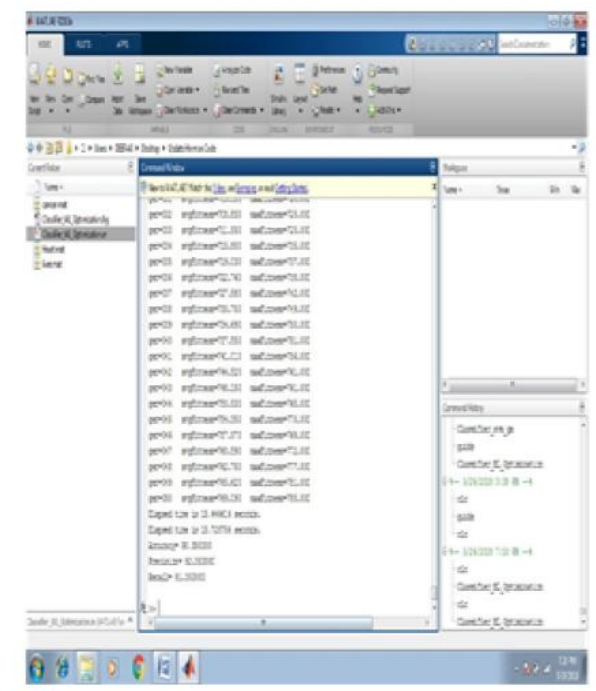

Figure-6: This windows show that the result of Proposed methods with accuracy in the experimental process using Liver dataset. 


\section{Comparatice Result Analysis of Heart Diseases Dataset using Machine Learning and Optimization Method}

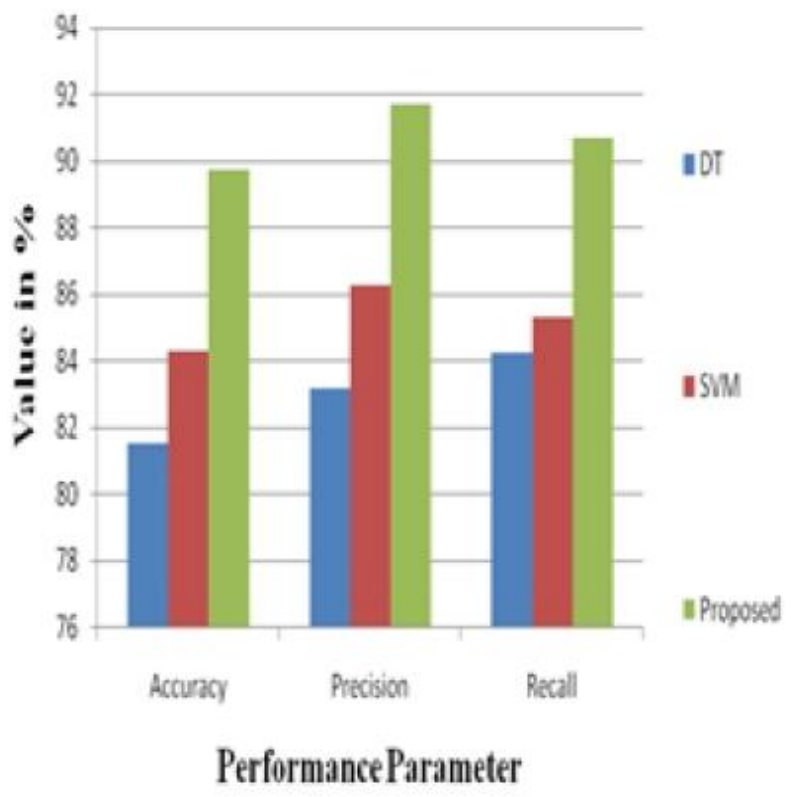

Figure 7: Figure show that the comparative result analysis for the Heart disease diagnosis.

\section{CONCLUSIONS AND FUTURE WORK}

In this paper we focus on pattern extraction and pattern analysis of healthcare data environment using particle of swarm optimization, the combination of support vector machines and particle of swarm optimization gives us better results than previous implemented techniques which is a combination of feed forward neural network and particle of swarm optimization. The diversity of medical diagnosis of disease data are increase day to day. Now in future dimension reduction process are also involved in ensemble classification technique.

\section{ACKNOWLEDGMENT}

Firstly, I would like to express my sincere gratitude to my advisor Prof. Imran Ali Khan for the continuous support of my M.Tech study and related research, for his patience, motivation, and immense knowledge. His guidance helped me in all the time of research and writing of this thesis. I could not have imagined having a better advisor and mentor for my M.Tech study.

Besides my advisor, I would like to thank the rest of my thesis committee: Prof. Vinod Kumar Yadav, Prof. Anuj Pal, and Prof. Pushpraj, for their insightful comments and encouragement, but also for the hard question which incented me to widen my research from various perspectives.

My sincere thanks to Dr. Aditya Vidhyarthi, Dr. Shishir Kumar Shandilya, who provided me an opportunity to join their team as intern, and who gave access to the laboratory and research facilities. Without their precious support it would not be possible to conduct this research.

I thank my fellow labmates in for the stimulating discussions, for the sleepless nights we were working together before deadlines, and for all the fun we have had in the last two years. Also I thank my friends in the following institution Sumit Jaiswal. In particular, I am grateful to Prof. Shobhana Shimoun Patras for enlightening me the first glance of research.
Last but not the least, I would like to thank my family: my parents and to my brothers and sister for supporting me spiritually throughout writing this thesis and my my life in general.

\section{REFERENCES}

[1] Min Chen, Yixue Hao, Kai Hwang, Lu Wang, And Lin Wang, "Disease Prediction by Machine Learning Over Big Data From Healthcare Communities" Special Section On Healthcare Big Data, Vol-5, IEEE, 2017. Pp 8869-8879.

[2] Sudha Ram, Wenli Zhang, Max Williams, Yolande Pengetnze, "Predicting Asthma-Related Emergency Department Visits Using Big Data", IEEE Journal of Biomedical and Health Informatics, vol. 19, 2015. Pp 1216-1218.

[3] Marco Viceconti, Peter Hunter, and Rod Hose, "Big Data, Big Knowledge: Big Data for Personalized. Healthcare", IEEE JOURNAL OF BIOMEDICAL AND HEALTH INFORMATICS, VOL. 19,, 2015. Pp 1209-1215.

[4] Javier Andreu-Perez, Carmen C. Y. Poon, Robert D. Merrifield, Stephen T. C. Wong, and Guang-Zhong Yang," Big Data for Health", IEEE Journal of Biomedical And Health InformaticS, VOL. 19, 2015. Pp 1193-1206.

[5] Yin Zhang, Meikang Qiu, Chun-Wei Tsai, Mohammad Mehedi Hassan, Atif Alamri, "Health-CPS: Healthcare Cyber-Physical System Assisted by Cloud and Big Data", IEEE Systems Journal, 2015. Pp 1-9.

[6] Ashwin Belle, Raghuram Thiagarajan, S. M. Reza Soroushmehr, Fatemeh Navidi, Daniel A. Beard, Kayvan Najarian, "Big Data Analytics in Healthcare", Hindawi Publishing Corporation Bio-Medical Research International, 2015. Pp 1-17.

[7] Wullianallur Raghupathi, Viju Raghupathi, "Big data analytics in healthcare: promise and Potential", Raghupathi and Raghupathi Health Information Science and Systems 2014. Pp 1-10.

[8] Michael K. K. Leung, Andrew Delong, Babak Alipanahi, Brendan J. Frey, "Machine Learning in Genomic Medicine: A Review of Computational Problems and Data Sets", IEEE Vol-104, 2016. Pp 176-197.

[9] Daniele Rav1, Charence Wong, Fani Deligianni, Melissa Berthelot, Javier Andreu-Perez, Benny Lo, Guang-Zhong Yang," Deep Learning for Health Informatics", Ieee Journal Of Biomedical and Health Informatics, VOL. 21, 2017. Pp 4-21.

[10] Michael J. Paul, Abeed Sarker, John s. Brownstein, Azadeh Nikfarjam, Matthew Scotch, Karen 1. Smith, Graciela Gonzalez, "social media mining for public health monitoring and surveillance", Pacific Symposium on Biocomputing 2016. Pp 468-477.

[11] Zoubin Ghahramani, "Probabilistic machine learning and arti_cial intelligence”, 2015. Pp 1-24.

[12] Ruogu Fang, Samira Pouyanfar, Yimin Yang, Shu-Ching Chen, S. S. Iyengar, "Computational Health Informatics in the Big Data Age: A Survey", ACM Computing Surveys, Vol. 49, 2016. Pp 1-36.

[13] Gunasekaran Manogaran, Chandu Thota, Daphne Lopez, V. Vijayakumar, Kaja M. Abbas and Revathi Sundarsekar, "Big Data Knowledge System in Healthcare", 2017. Pp 133-158. 
[14] Rahul C. Deo, "Machine Learning in Medicine", 2017. Pp 1920-1931.

[15] Riccardo Miotto, Fei Wang, Shuang Wang, Xiaoqian Jiang, Joel T. Dudley, "Deep learning for healthcare: review, opportunities and Challenges", Briefings in Bioinformatics, 2017, Pp 1-11.

[16] Ji-Jiang Yang, Jianqiang Li, Jacob Mulder, Yongcai
Wang, Shi Chen, Hong Wu, Qing Wang, Hui Pan, "Emerging information technologies for enhanced healthcare', Elsevier ltd. 2015. Pp 3-11.

[17] Majid Ghonji Feshki and Omid Sojoodi Shijani "Improving the Heart Disease Diagnosis by Evolutionary Algorithm of PSO and Feed Forward Neural Network", IEEE, 2016, Pp 48-53. 